\title{
Caracterização química e atividade biológica de extratos aquosos de Brunfelsia cuneifolia J.A. Schmidt (Solanaceae)
}

SCHNEIDER, A.L.S.'; BERTELLI, P.R. '; BARRETO, M.L.'; ABREU, N.V.'; AGOSTINI, F.'; SCHWAMBACH, J."* 'Universidade de Caxias do Sul, Rua Francisco Getúlio Vargas, 1130, CEP 95070-560, Caxias do Sul - RS Brasil, *Autor para correspondência: joselischwambach@gmail.com

\begin{abstract}
RESUMO: O gênero Brunfelsia possui ainda poucas informações a respeito de sua composição química ou confirmações científicas de suas propriedades medicinais, apesar do uso na medicina tradicional pelos povos amazônicos. Este trabalho buscou caracterizar a espécie Brunfelsia cuneifolia, cultivada no estado do Rio Grande do Sul, quanto a sua composição química e atividade biológica. Foram obtidos extratos aquosos a quente, a frio, e por ultrassom, a partir de folhas frescas. A caracterização química realizada por CLAE determinou a presença dos compostos fenólicos: ácido ferúlico e rutina, em todos os extratos, sendo as maiores quantidades apresentadas pela extração a frio. A análise por EMAR identificou a fórmula molecular de nove substâncias nos diferentes extratos, incluindo a presença do alcaloide brunfelsamidina em todos os extratos obtidos. Para a atividade biológica, devido à similaridade de resposta e teor nas diferentes formas de extração, foi possível correlacionar a atividade antioxidante, avaliada através da redução do radical $\mathrm{DPPH}^{*}$, com o teor de compostos fenólicos totais obtidos pelo método de Folin-Ciocalteu. A toxicidade dos extratos avaliada pela utilização de Artemia salina revelou ausência de toxidez. Os resultados obtidos são os primeiros apresentados para a caracterização desta espécie, colaborando também para a pesquisa científica acerca dos usos popularmente atribuídos ao gênero.
\end{abstract}

Palavras-chave: manacá, antioxidantes, compostos fenólicos, brunfelsamidina, toxicidade.

\begin{abstract}
Preliminary chemical characterization and biological activity of aqueous extracts of Brunfelsia cuneifolia J.A. Schmidt (Solanaceae). There is still not a lot of information about the Brunfelsia genus. Its respective chemical composition and possible scientific proofs of its medicinal properties were not deeply researched, even though the use of the species is common in the traditional medicine of Amazonian populations. This study aimed to characterize the Brunfelsia cuneifolia species, grown in the state of Rio Grande do Sul, regarding its chemical composition and biological activity. Hot, cold and ultrasound aqueous extracts of fresh leaves were obtained. The chemical characterization performed by the HPLC determined the presence of phenolic compounds, ferulic acid and rutin in all extracts, with the largest amount presented by the cold extraction method. The HRMS analysis identified the molecular formula of nine substances in different extracts, including the presence of the alkaloid brunfelsamidina in all the extracts. For biological activity, it was possible to correlate the measured antioxidant activity through the reduction of DPPH * with the content of phenolic compounds obtained by the Folin-Ciocalteu method, which exhibited similar response and content in the different forms of extraction. The toxicity of the extracts evaluated through the use of Artemia salina was negative. The obtained results are the first ones presented for the characterization of this species, also contributing to scientific research on the popular applications related to this genus.
\end{abstract}

Keywords: yesterday-today-tomorow, antioxidant, phenolic compounds, brunfelsamidine, toxicity.

\section{INTRODUÇÃO}

A família Solanaceae A. Jussieu é uma das maiores das angiospermas, compreendendo cerca de 2.300 espécies subordinadas a 92 gêneros amplamente distribuídos em regiões tropicais e subtropicais, tendo a América do Sul como um dos seus principais centros de diversidade e endemismo 
(Hunziker, 2001). Possui reconhecida importância econômica por conter espécies com atribuições ornamentais, medicinais e nutricionais (Agra \& Bhattacharyya, 1999; Silva et al., 2005).

Pertencente a esta família, o gênero Brunfelsia L. compreende espécies conhecidas popularmente por manacá-de-cheiro ou primavera (Longhi, 1995), manacá, manacá-açu, manacá-daserra (Pio Corrêa, 1974; Backes \& Nardino, 2003). Exuberantes, espécies deste gênero constam em catálogos de plantas ornamentais (Lorenzi \& Souza, 2001). Xamãs e curandeiros da região amazônica atribuem poderes mágicos ao gênero, utilizando a planta em rituais sagrados (Plowman, 1977). Levantamentos etnobotânicos relatam o uso de espécies de Brunfelsia como antirreumático (B. chiricaspi Plowman, B. uniflora (Pohl) D. Don e B. guianenses Benth.), emético (B.uniflora), antissifilítico (B. guianenses e B. uniflora), depurativo (B. guianenses), diurético (B. uniflora), antidiarréico (B. americana L.), vermífugo (B. mire Monach.), purgativo (B.uniflora), entre outros (Schultes, 1979).

Outras espécies do gênero são suspeitas de conterem atividade farmacológica ou de possuírem flavonóides, alcaloides ou outros constituintes ativos (Plowman, 1977; Brunner et al., 2000). Química e farmacologicamente, as espécies de Brunfelsia são ainda pouco conhecidas, o que é instigante considerando as muitas contribuições que a família Solanaceae proporcionou a medicina atual (Plowman, 1977). Ainda assim, $B$. uniflora consta na primeira edição da Farmacopéia Brasileira (Rodolpho, 1929) e na terceira edição do PDR for Herbal Medicines (Gruenwald et al., 2000).

No Rio Grande do Sul, a espécie Brunfelsia cuneifolia J.A. Schmidt é cultivada com frequência em locais de domínio público ou privado (Soares \& Mentz, 2007). Esta espécie caracteriza-se por apresentar flores com um cálice florífero tubuloso, visivelmente anguloso em exemplares vivos e plicados em material herborizado. Para sua ocorrência natural, há registro nas regiões fisiográficas da Encosta Superior do Nordeste, Campos de Cima da Serra e Planalto Médio. Habita o interior das matas, tendo sido encontrada somente em Floresta Ombrófila Mista, às vezes formando comunidades com Brunfelsia pilosa (Soares \& Mentz, 2007).

Devido à ausência de estudos fitoquímicos e biológicos sobre Brunfelsia cuneifolia, este trabalho visou avaliar o potencial antioxidante e a toxicidade de diferentes extratos obtidos de folhas desta espécie, bem como identificar suas composições químicas via cromatografia líquida de alta eficiência (CLAE) e espectrometria de massas de alta resolução (EMAR).

\section{MATERIAIS E MÉTODOS \\ Material vegetal}

As partes aéreas de diferentes indivíduos de B. cuneifoólia J.A. Schmidt foram coletadas no município de Caxias do Sul (28'19'27.474"S / $51^{\circ} 11^{\prime} 42.424^{\prime \prime} W$ ) em setembro de 2012. Exsicatas foram depositadas no herbário da Universidade de Caxias do Sul, sob o número HUCS 40384, e foram identificadas por Edson Luís de Carvalho Soares.

A espécie vegetal possui Autorização Especial de Acesso e Remessa de Amostra de Componente do Patrimônio Genético junto ao CGAUF/IBAMA com o titulo de "Plantas nativas do Rio Grande do Sul com potencial efeito fitoterápico e aplicação em controle biológico" n 02001.004237/2013-16.

\section{Preparação dos extratos}

Extrações aquosas utilizando $5 \%(\mathrm{p} / \mathrm{v}) \mathrm{de}$ folhas frescas rasuradas foram realizadas através de três métodos: a quente, temperatura ambiente (frio) e ultrassom. A extração a quente foi realizada através da infusão das folhas, juntando-se água destilada fervente sobre planta, cobrindo o becker e deixando-se em repouso por 10 minutos (Lorenzi \& Matos, 2002). Na extração a frio, a planta foi imersa em água destilada a temperatura ambiente, permanecendo durante 12 horas (Lorenzi \& Matos, 2002). Para a extração por ultrassom, a planta juntamente com água destilada foi sonicada por 10 minutos em banho ultrassônico sem aquecimento Quimis ${ }^{\circledR}$ (Modelo Q335D) com 135 Watts RMS (Carrera et al., 2012). Todos os extratos foram filtrados em papel filtro sob pressão reduzida.

\section{Determinação de compostos fenólicos totais no extrato}

A quantificação de compostos fenólicos foi realizada de acordo com Roesler et al. (2007) pelo método espectrofotométrico de Folin-Ciocalteu. A leitura foi realizada em espectrofotômetro (DU530 - Beckman) a 760 nm. O conteúdo de compostos fenólicos totais foi quantificado utilizando uma curva padrão preparada com ácido gálico (10-120 $\mu \mathrm{g} / \mathrm{mL}$; Chem Service, Inc., USA), e o resultado expresso como microgramas de equivalentes de ácido gálico por mililitros de extrato (ug EAG/mL EXT).

\section{Determinação da capacidade antioxidante - método DPPH}

A atividade de sequestro do radical DPPH* (2,2 difenil-1-picrilhidrazil) foi quantificada utilizando a metodologia de Chang et al. (2007), onde diferentes concentrações de extrato $(5,4,3,2$ e $1 \%)$ foram adicionados ao tampão 100nM TRIS- $\mathrm{HCl}(\mathrm{pH}$ $7,4)$ e a solução de $500 \mu \mathrm{M}$ de DPPH em etanol.

Rev. Bras. PI. Med., Campinas, v.17, n.4, supl. III, p.1103-1111, 2015. 
A mistura foi deixada em repouso no escuro por 20 minutos. A absorbância foi medida em $517 \mathrm{~nm}$ no espectrofotômetro de microplaca (SpectraMax M2 ${ }^{\mathrm{e}}$ - Molecular Devices). A análise foi realizada em triplicata. A percentagem de descoloração de DPPH da amostra foi calculada de acordo com a seguinte equação: \% descoloração $=[1-\mathrm{ABS}$ amostra / ABS controle] $\times 100$. O $\mathrm{CE}_{50}$ é a concentração eficaz no qual $50 \%$ dos radicais DPPH foram sequestrados nas condições do ensaio, e foi obtido por interpolação a partir da análise de regressão linear. O resultado foi multiplicado por 100 para ser expresso em porcentagem.

\section{Avaliação de toxicidade frente a larvas de Artemia salina}

A avaliação da toxicidade frente à $A$. salina foi realizada segundo metodologia descrita por McLaughlin et al. (1998), onde $10 \mathrm{mg}$ de ovos do microcrustáceo adicionados a uma solução artificial de água do mar $(26,30 \mathrm{~g} / \mathrm{L} \mathrm{NaCl}, 0,75 \mathrm{~g} / \mathrm{L} \mathrm{KCl}, 1,11$ $\mathrm{g} / \mathrm{L} \mathrm{CaCl}_{2}, 5,10 \mathrm{~g} / \mathrm{L} \mathrm{MgCl}{ }_{2}, 0,08 \mathrm{~g} / \mathrm{L} \mathrm{NaBr}, 0,21 \mathrm{~g} / \mathrm{L}$ $\mathrm{NaHCO}_{3}$ e 6,20 $\mathrm{g} / \mathrm{L} \mathrm{MgSO}_{4}$ ) foram expostos à luz artificial, aeração e temperatura de $25-30^{\circ} \mathrm{C}$ durante 48 horas para a eclosão dos ovos. O ensaio foi realizado utilizando placas de 24 poços, onde $500 \mu \mathrm{L}$ de cada extrato foi previamente evaporado à secura. A cada poço contendo o extrato evaporado, foram adicionados $400 \mu \mathrm{L}$ de solução artificial de água do mar e transferidos 10-15 náuplios contidos em 100 $\mu \mathrm{L}$ da solução inicial de água do mar. O controle foi realizado nas mesmas condições, sem a presença dos extratos. As placas tampadas foram mantidas sob luz artificial e temperatura de $25-30^{\circ} \mathrm{C}$ por 24 horas, quando então foi realizada a contagem dos sobreviventes do grupo controle e dos grupos expostos aos extratos, sendo então determinando o percentual de morte do grupo teste e controle através de percentagem simples. O experimento foi realizado em triplicata.

\section{(CLAE)}

Análises químicas Cromatografia líquida de Alta Eficiência

A identificação e quantificação dos compostos fenólicos presentes nos extratos foi baseada no método de Fillip et al. (2001), utilizandose equipamento marca HP modelo 1100 , coluna Lichrospher $\operatorname{RP}_{18}(5 \mu \mathrm{m})$ equipado com detector UV a $350 \mathrm{~nm}$ e sistema quaternário de bombas. A fase móvel foi constituída de: Metanol com $2 \%$ de ácido acético (eluente $A$ ) e água ultrapura Milli $Q^{\circledR} \operatorname{com} 2 \%$ de ácido acético (eluente $B$ ) em sistema gradiente de bombeamento de fase móvel ( 0 a 30 min de 15 a $40 \%$ de A, 40 min $75 \%$ de A, 45 a 50 min $85 \%$ de A). O tempo total de análise foi de 50 minutos e o fluxo padrão foi mantido a $1,0 \mathrm{~mL} / \mathrm{min}$. Os extratos foram solubilizados em metanol $(1 \mathrm{~g} / \mathrm{L})$ e filtrados em membranas de Nylon de 0,45 $\mu \mathrm{m}$ de diâmetro de poro. Uma alíquota de $50 \mu \mathrm{L}$ desta solução foi injetada no cromatógrafo. Quercetina, canferol, ácido clorogênico, apigenina, luteolina, naringina, rutina e ácido ferúlico foram utilizados como padrões de referência. (EMAR)

Espectrometria de Massas de Alta Resolução

Os espectros foram obtidos em espectrômetro de massas microTOF-Q II (Q-TOF) (Bruker Scientific ${ }^{\circledR}$ ) no modo positivo (ESI(+)-MS por infusão direta, sob as seguintes condições: tensões capilares e cone foram definidos em $+3500 \mathrm{~V} \mathrm{e}+40$ $\mathrm{V}$, respectivamente, com temperatura de solvatação de $100^{\circ} \mathrm{C}$. Quinze microlitros dos extratos obtidos pelos três métodos extrativos propostos foram solubilizados em metanol grau HPLC e $30 \mu \mathrm{L}$ de água ultrapura Milli $Q^{\circledR}$ foram adicionados. As soluções foram infundidas diretamente na fonte ESI por meio de seringa (Harvard Apparatus) em injetor automático, a uma taxa de fluxo de $10 \mu \mathrm{L} \mathrm{min} \mathrm{m}^{-1}$. Para a aquisição e processamento de dados obtidos no analisador de massas (Q-TOF) um software de análise de dados (Data Analysis - Bruker ${ }^{\circledR}$ Scientific) foi utilizado. $O$ equipamento foi programado para monitorar os íons na faixa $\mathrm{m} / \mathrm{z}$ 90-1000.

\section{Análise estatística}

Os dados obtidos foram submetidos à análise estatística por Análise de Variância (ANOVA) seguida de teste de Tukey, utilizando programa de estatística SPSS 18.0 for Windows.

\section{RESULTADOS E DISCUSSÃO}

O emprego da planta medicinal fresca, recém-colhida ou elaborada extemporaneamente, mesmo que de maneira empírica, é o recurso terapêutico mais frequentemente utilizado pela maior parte da população brasileira, especialmente no Nordeste e Região Amazônica (Lorenzi \& Matos, 2002). Assim, neste trabalho buscou-se testar as formas mais comuns de extração de princípios ativos vegetais: extratos aquosos das folhas frescas, a quente e a frio. Como comparativo aos processos estáticos, utilizou-se a extração por ultrassom em água a temperatura ambiente.

Amplamente distribuídos no reino vegetal, a classe de compostos fenólicos típicos inclui os derivados do ácido benzóico (ex. ácido gálico) e do ácido cinâmico (ex. ácido caféico, acido clorogênico e ácido ferúlico), cumarinas (ex. escopoletina), flavonoides (ex. quercetina e rutina) e derivados de polimerização (taninos e ligninas). São moléculas heterogêneas que apresentam grande variação

Rev. Bras. PI. Med., Campinas, v.17, n.4, supl. III, p.1103-1111, 2015. 
estrutural, possuindo pelo menos um anel aromático no qual, ao menos, um hidrogênio é substituído por um grupamento hidroxila (Simões et al., 2003). Compostos desta classe possuem reconhecida atividade antioxidante, resultado de um conjunto de propriedades inerentes à sua estrutura química, além de atividades anti-inflamatórias, antivirais, antitumorais, hormonais, antiespasmódicas e antimicrobianas, entre outras (Simões et al. 2003).

A presença de compostos fenólicos em $B$. cuneifolia foi investigada. A quantificação destes através do método de Folin-Ciocalteu demonstrou que foram obtidos derivados fenólicos em todos os métodos extrativos empregados. (Figura 1).

$O$ radical DPPH tem sido amplamente utilizado como um sistema modelo para investigar a atividade antioxidante de vários compostos naturais, incluindo compostos fenólicos isolados, flavonoides, ou misturas brutas, tais como extratos etanólicos e aquosos de plantas (Roginsky \& Lissi, 2005). No presente estudo, todas as amostras, independentemente do método de extração, apresentaram atividade antioxidante dosedependente. A determinação da $\mathrm{CE}_{50}$ revela que, apesar de não haver diferença estatística entre os extratos analisados, há uma tendência no extrato frio em apresentar uma maior capacidade antioxidante (Figura 2).

Observa-se que métodos aquosos simples de extração, similares aos utilizados popularmente, permitem a extração de derivados fenólicos com ação antioxidante no material estudado. A relação entre o potencial antioxidante dos extratos e o teor de compostos fenólicos totais mostrou-se diretamente proporcional, o que corrobora muitos estudos na área (Soares et al., 2003; Spada \& Salvador, 2005). Para o gênero Brunfelsia, este comportamento de correlação foi também encontrado na avaliação do extrato metanólico de folhas de $B$. americana, aferindo para a espécie significativa atividade antioxidante em comparação ao ácido ascórbico (Raj et al, 2010). Cabe salientar que outras espécies da família Solanaceae apresentam atividade antioxidante mais efetiva, devido às menores concentrações necessárias para desempenhar tal atividade como, por exemplo: Solanum paniculatum (Ribeiro et al., 2007) e Physalis peruviana L. (Rockenback et al., 2008).

A análise química dos extratos realizada por cromatografia liquida revelou a presença de dois compostos fenólicos majoritários: rutina e acido ferúlico (Figura 3). Dos padrões testados, não foi possível identificar a presença de quercetina, canferol, ácido clorogênico, apigenina, luteolina e naringina em nenhum extrato avaliado. Com relação à rutina e ácido ferúlico, ambos compostos fenólicos foram encontrados em maior concentração no extrato aquoso frio, o que pode justificar a tendência à maior atividade antioxidante deste extrato. A rutina é um glicosídeo conjugado pertencente à classe dos flavonóis, com propriedades farmacológicas como ação antioxidante, citoprotetora, vasoprotetora e cardioprotetora, entre outras (Araujo, 2012). Estudos realizados com diferentes flavonoides, incluindo rutina, apontam para seu elevado potencial antioxidante, inclusive quando comparado à vitamina C (Bianchi \& Antunes, 1999). Já o ácido ferúlico é um derivado do ácido cinâmico, com indicações para uso em tratamento e prevenção de câncer e doenças cardiovasculares, entre outras. Investigações

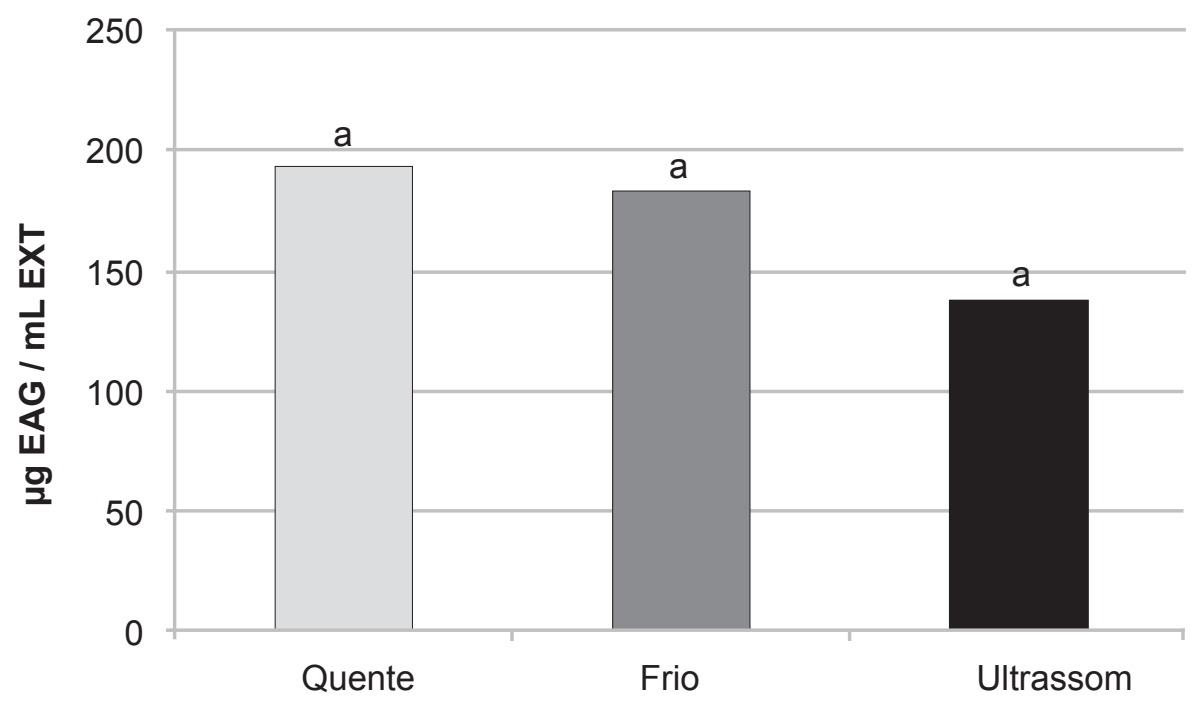

FIGURA 1. Teor de compostos fenólicos totais em extratos aquosos de $B$. cuneifolia obtidos por infusão a quente, a frio e por ultrassom. Colunas com letras iguais não diferem significativamente pela Análise de Variância $(p \leq$ $0,05)$. 


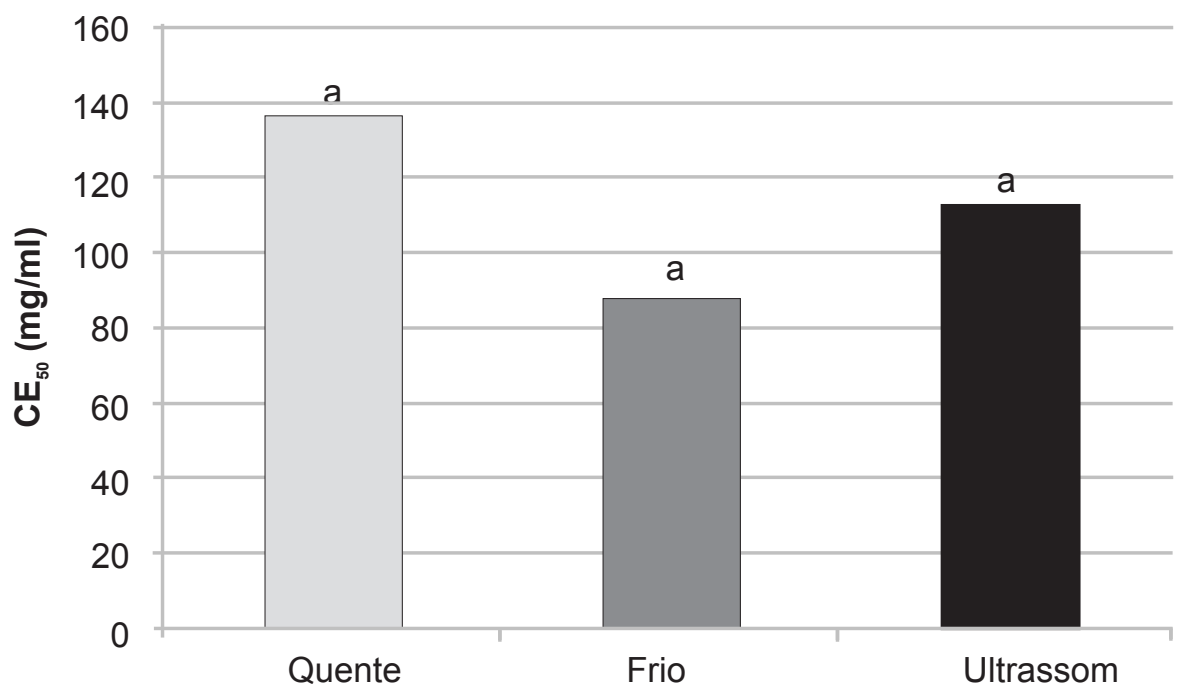

FIGURA 2. Atividade antioxidante dos extratos aquosos de $B$. cuneifolia obtidos por infusão a quente, a frio e por ultrassom. $\mathrm{CE}_{50}$ representa a concentração eficaz para reduzir $50 \%$ do racical $\mathrm{DPPH}^{*}$. Colunas com letras iguais não diferem significativamente pela Análise de Variância $(p \leq 0,05)$.

realizadas com ácidos fenólicos, incluindo ácido ferúlico, demonstram atividade antioxidante significativa deste composto quando comparado aos ácidos clorogênico, cafeico e p-cumárico (Soares, 2002). Em Brunfelsia, a cumarina escopoletina encontrada em $B$. uniflora e B. grandiflora (Mors \& Robeiro, 1957; lyer et al., 1977; Oliveira et al., 2000; Fuchino et al., 2008), possui ação espasmolítica (Oliveria et al., 2000) e é um importante constituinte observado em plantas utilizadas contra veneno de cobra (Mors et al., 2000). Em B. grandiflora, Brunner et al. (2000) identificaram a estrutura química de um flavonóide isolado da espécie. Martins et al. (2009) sinalizaram a ocorrência de derivados do ácido cafeico em extratos de $B$. uniflora, sugerindo para a espécie a presença de ácido clorogênico. Desta forma, o resultado apresentado em $B$. cuneifolia corrobora a informação da presença de flavonoides e ácidos fenólicos derivados do ácido cinâmico em espécies de Brunfelsia.

Existem poucas informações a respeito da constituição química do gênero Brunfelsia, e não existem estudos na área envolvendo Brunfelsia cuneifolia. A presença do alcaloide hopeamina (lyer 1978) e da cumarina escopoletina (lyer et al., 1977) a partir de extratos das raízes de $B$. uniflora (sinônimo de B. hopeana) foi relatada. Birkner et al. (1986) obtiveram o alcaloide brunfelsamidina das folhas, caules e das raízes, da mesma espécie.

Para amostras complexas, como extratos

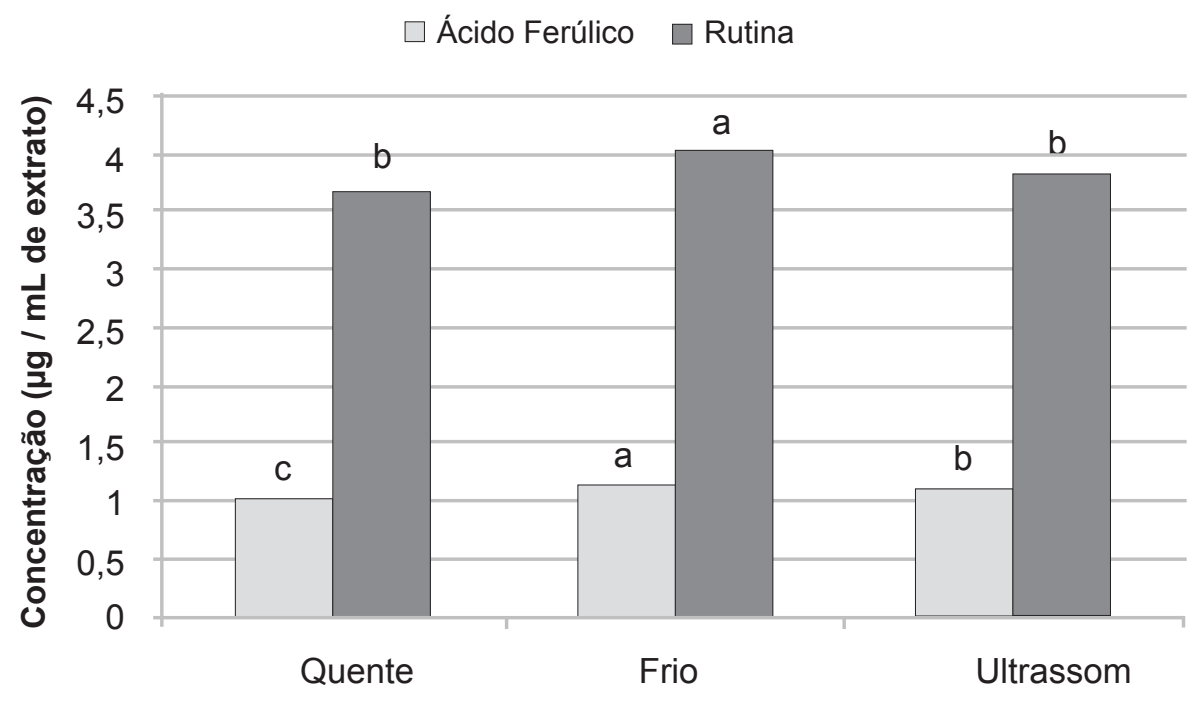

FIGURA 3. Concentração dos compostos fenólicos majoritários encontrados nos extratos aquosos de B. cuneifolia obtidos por infusão a quente, a frio e por ultrassom. Colunas com letras iguais não diferem significativamente pela Análise de Variância $(p \leq 0,05)$. 
de plantas, faz-se necessário a utilização de instrumentação robusta, sensível e com boa resolução, capaz de gerar informações químicas confiáveis. Deste modo, a Espectrometria de Massas de Alta Resolução (EMAR) foi eleita para a realização de um screening prévio na espécie sob estudo. A análise de massas tem tido êxito na qualificação dos componentes em misturas de extratos de plantas, fornecendo dados sobre o peso e a fórmula molecular e possibilitando a identificação de fragmentos característicos da molécula (Maciel et al., 2002). A confirmação da presença de compostos químicos é possível devido à associação de informações obtidas pelo equipamento, como a acurácia nas medidas das razões massa/carga $(\mathrm{m} / \mathrm{z})$, razão isotópica e fragmentação de moléculas.

Neste estudo, foram avaliados os três extratos aquosos. Nos espectros de EMAR apresentados na Figura 4, podemos visualizar que os extratos a quente (A) e a frio (B), apesar de se diferenciarem entre si pelo tempo e temperatura de extração, apresentam perfis químico muito semelhantes, com moléculas com $\mathrm{m} / \mathrm{z}$ entre 100 200. No entanto, o perfil apresentado pela extração através do método de ultrassom acusou a presença de moléculas com maior massa molecular (C). . Esta diferença pode estar relacionada ao fenômeno de cavitação inerente ao processo físico ultrassônico, que facilita a ruptura de partículas, a extração de compostos e a ativação de reações químicas (Emídio \& Dórea, 2010).

A partir das informações obtidas no EMAR, como a massa exata e razão isotópica, foi determinada a composição elementar dos principais picos apresentados nos espectros. Para a acurácia, uma diferença não superior a 5 ppm entre o experimental e o teórico seria o indicado para confirmar a identificação de moléculas-alvo (Lacorte \& Fernandez-Alba, 2006). Na Tabela 1, encontramse as 9 fórmulas moleculares identificadas nos extratos aquosos analisados.

Devido aos escassos estudos químicos
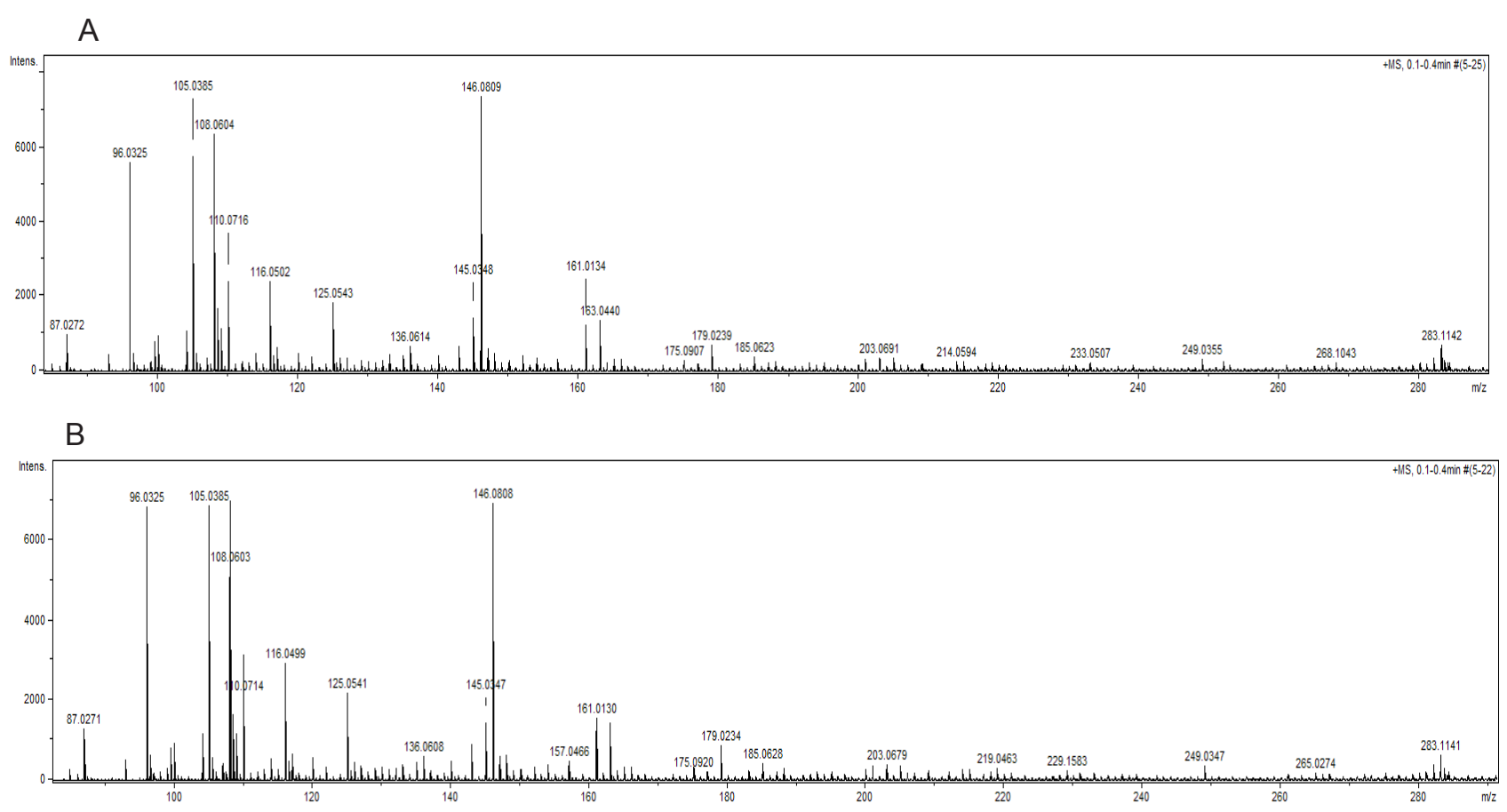

C

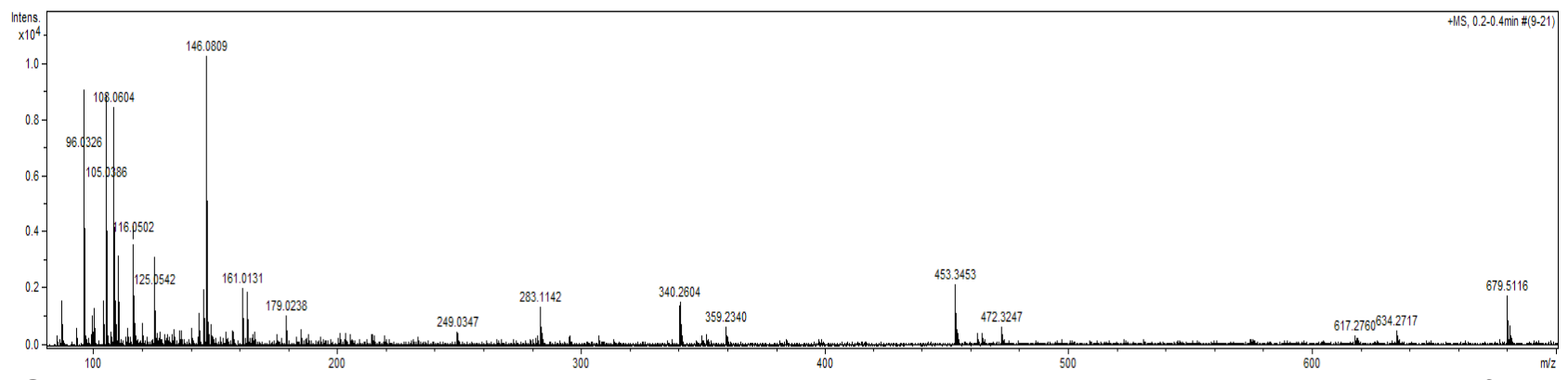

FIGURA 4. Espectros de massas de alta resolução no modo positivo dos extratos aquosos de Brunfelsia cuneifolia: A) Extração com água a 100 •C (quente); B) Extração com água a temperatura ambiente (frio); C) Extração com água em ultrassom.

Rev. Bras. PI. Med., Campinas, v.17, n.4, supl. III, p.1103-1111, 2015. 
envolvendo o gênero Brunfelsia, apenas as fórmulas moleculares puderam ser identificadas. No entanto, a fragmentação destes compostos pode contribuir com importante informação. No espectro, apresentado no modo MS-2 da Figura 5, observamos o pico referente ao íon molecular com $\mathrm{m} / \mathrm{z} 110.0681$ e de sua fragmentação com $\mathrm{m} / \mathrm{z}$ 93.0408, devido a perda do fragmento $\mathrm{NH}_{2}$, no momento da ionização na fonte onde o extrato foi infundido (Buschi et al. 1987). Esse padrão de fragmentação permite confirmar a presença de brunfelsamidina $(1 \mathrm{H}$ Pyrrole-3-carboximidamide) em todos os extratos avaliados. Este composto foi encontrado por Lloyd et al. (1985) em Brunfelsia gradiflora e agora confirmamos a presença do mesmo composto em Brunfelsia cuneifolia, ampliando o conhecimento sobre a composição química do gênero.

Considerando o emprego do gênero Brunfelsia por algumas populações (Plowman, 1977) e os relatos publicados de que espécies do gênero apresentaram efeitos tóxicos quando ingeridas pelo gado (Spainhour et al., 1990), foi aplicado o teste de toxicidade utilizando larvas de Artemia salina. Este microcrustáceo de água salgada é muito sensível a substâncias tóxicas, e o teste é uma forma simples para avaliação de extratos de plantas e produtos químicos. $O$ teste pode também indicar a predisposição de atividade antitumoral ou pesticida de compostos (Khafagi et al., 2000). A relação entre larvas mortas (sem mobilidade) e larvas vivas (alta mobilidade) em comparação com o controle, sem nenhuma substância aplicada, é utilizada para estimar a toxicidade das soluções testadas. No teste realizado, não houve diferença estatística entre o grupo controle $(2,08 \%$ de mortalidade) e os extratos aquosos obtidos, ainda que a maior mortalidade tenha sido observada na extração a quente (10,3\%). Queiroz (2009) ao avaliar a toxicidade de extrato hidroetanolico de $B$. uniflora e suas frações utilizando $A$. salina observou que nenhuma das amostras apresentou toxicidade. Porém, Moreno-Murillo et al. (2001) encontrou atividade tóxica frente $A$. salina quando testaram o extrato alcoólico de B. pauciflora. Desta forma, estudos complementares devem ser realizados para determinação da toxicidade relativa ao método extrativo empregado, uma vez que o resultado obtido não permitiu inferir toxicidade relevante para a espécie.

TABELA 1. Principais componentes químicos identificados por espectrometria de massas de alta resolução no modo positivo dos extratos aquosos de Brunfelsia cuneifolia. (A)- aquoso quente $\left(100^{\circ} \mathrm{C}\right)$ e frio $\left(25^{\circ} \mathrm{C}\right)$; $(\mathrm{U})$ - Ultrassom;

\begin{tabular}{lccccc}
\hline Entrada & $\begin{array}{c}\text { Íon precursor } m / z[M \\
+\mathrm{H}]^{+}\end{array}$ & $\begin{array}{c}\text { Razão isotópica } \\
{[\mathrm{M}+1+\mathrm{H}]^{+}(\%)}\end{array}$ & $\begin{array}{c}\text { Composição } \\
\text { elementar }\end{array}$ & $\begin{array}{c}\text { Diferença } \\
(\mathrm{ppm})\end{array}$ & Extratos \\
\hline 1 & 110.0714 & $111.0788(5.2)$ & $\mathrm{C}_{5} \mathrm{H}_{8} \mathrm{~N}_{3}$ & 3.8 & $(\mathrm{~A})(\mathrm{U})$ \\
2 & 116.0504 & $117.0535(8.2)$ & $\mathrm{C}_{8} \mathrm{H}_{6} \mathrm{~N}$ & 3.2 & $(\mathrm{~A})(\mathrm{U})$ \\
3 & 146.0812 & $147.0853(7.3)$ & $\mathrm{C}_{6} \mathrm{H}_{12} \mathrm{NO}_{3}$ & 3.5 & $(\mathrm{~A})(\mathrm{U})$ \\
4 & 283.1080 & $284.1178(15.3)$ & $\mathrm{C}_{14} \mathrm{H}_{13} \mathrm{~N}_{5} \mathrm{O}_{2}$ & 3.8 & $(\mathrm{~A})(\mathrm{U})$ \\
5 & 340.2594 & $341.2633(17.5)$ & $\mathrm{C}_{16} \mathrm{H}_{32} \mathrm{~N}_{6} \mathrm{O}_{2}$ & 2.1 & $(\mathrm{U})$ \\
6 & 453.3404 & $454.3411(18.3)$ & $\mathrm{C}_{17} \mathrm{H}_{43} \mathrm{~N}_{9} \mathrm{O}_{5}$ & 3.7 & $(\mathrm{U})$ \\
7 & 472.3271 & $473.3371(26.0)$ & $\mathrm{C}_{24} \mathrm{H}_{40} \mathrm{~N}_{8} \mathrm{O}_{2}$ & 0.7 & $(\mathrm{U})$ \\
8 & 679.5116 & $680.5092(38.4)$ & $\mathrm{C}_{36} \mathrm{H}_{67} \mathrm{~N}_{6} \mathrm{O}_{6}$ & 0.9 & $(\mathrm{U})$ \\
9 & 701.4915 & $702.4944(47.6)$ & $\mathrm{C}_{44} \mathrm{H}_{65} \mathrm{~N}_{3} \mathrm{O}_{5}$ & 3.1 & $(\mathrm{U})$ \\
\hline
\end{tabular}

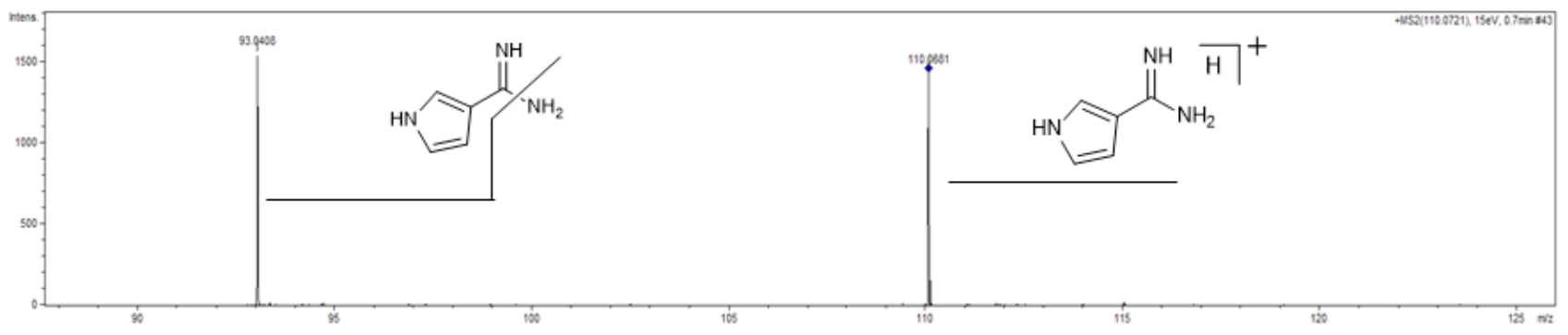

FIGURA 5. Espetro de massa de alta resolução MS2 em modo positivo dos extratos aquosos de Brunfelsia cuneifolia apresentando a fragmentação do íon molecular m/z 110.0714 correspondente a brunfelsamidina. 
Os resultados obtidos colaboram para a ampliação do conhecimento acerca do gênero Brunfelsia, apresentando a identificação dos compostos fenólicos rutina e ácido ferúlico, bem como a capacidade antioxidante dos extratos aquosos de $B$. cuneifolia. Da mesma forma, este é o primeiro estudo para o gênero utilizando a técnica de EMAR, o que tornou possível apontar a presença de compostos ainda não estudados e confirmar a presença do alcaloide brunfelsamidina nos extratos aquosos da espécie. Os estudos contribuem para o avanço da pesquisa sobre o gênero, na busca para o reconhecimento científico das propriedades popularmente atribuídas às espécies de Brunfelsia.

\section{REFERÊNCIAS}

AGRA, M.F; BHATTACHARYYA, J. Ethnomedicinal and phytochemical investigation of the Solanum species in the Northeast of Brazil. In: M. Nee, D.E. Symon, R.N.; Lester \& J. P. Jessop (ed.). Solanaceae IV: Advances in Biology and Utilization. Kew: Royal Botanic Gardens, 1999, p.341-343.

ARAUJO, M.E.M.B. Avaliação da atividade antioxidante e antiproliferativa da rutina e seus produtos obtidos por hidrolise enzimática, 2012, 78p. Dissertação ( Mestrado -Ciências da Saúde) Programa de PósGraduação Stricto Sensu em Ciências da Saúde, Universidade São Francisco, São Paulo.

BACKES, A.; NARDINO, M. Árvores, arbustos e algumas lianas nativas no Rio Grande do Sul. 2 ed. São Leopoldo: Editora Unisinos, 2003, 202p.

BIANCHI, M.L.P.; ANTUNES, L.M.G. Radicais livres e os principais antioxidantes da dieta. Revista de Nutrição, v. 12, n.2, p. 123-130, 1999.

BIRKNER, C. et al. Extraction of hopamidine. Patent-Ger. Offen-3,506,643; 12 pp, 1986.

BRUNNER, G. et al. A novel acylated flavonol glycoside isolated from Brunfelsia grandiflora ssp. structure elucidation by gradient accelerated NMR Spectroscopy at 14t. Phytochemical Analysis, v. 11, p.29-33, 2000..

BUSCHI, C.A.; POMILUO, A.B. Pyrrole-3-carbamidine: A lethal principle from Nierembergia hippomanica. Phytochemistry, v. 26, n. 3, p. 863-865, 1987.

CARRERA, C. et al. Ultrasound assisted extraction of phenolic compounds from grapes. Analytica Chimica Acta v. 732, p. 100-104, 2012.

CHANG, H. et al. Antioxidant activities and polyphenol contents of six folk medicinal ferns used as "Gusuibu". Botanical Studies, v. 48, p. 397-406, 2007.

EMÍDIO, E.S.; DÓREA, H.S.; Comparação entre as extrações ultra-som e Soxhlet para determinação de alcanos em sedimentos ambientais; Scientia Plena, v. 6, n. 9, 2010.

FILLIP, R., et al. Phenolic compounds in seven South American Ilex species. Fitoterapia, n.72 p.774-778, 2001.

FUCHINO, $\mathrm{H}$ et al. A new leishmanicidal saponin from Brunfelsia grandiflora. Chemical and Pharmaceutical Bulletin, v.56, p. 93-96, 2008.

GRUENWALD, J. (ed). Physician's desk reference (pdr) for herbal medicines, 3 ed. Montvale: NJ :Medical Economics Co., Inc., , 2000.1100p.

HUNZIKER, A.T. Genera Solanacearum: the genera of Solanaceae illustrated, arranged according to a new system. ARG Gantner Verlag KG: Ruggell, Liechenstein. 2001.500 p.

IYER, R.P. Brunfelsia hopeana - pharmacologic screening: isolation and characterization of hopeanine. University of Pacific, Stockton, Estados Unidos, 1978. 236p.

IYER, R.P. et al. Brunfelsia hopeana. I: Hippocratic screening and antiinflammatory evaluation. Lloydia, v.40, n.4, p.356-60, 1977.

KHAFAGI,I. et al. In vitro cytotoxicity and antimicrobial activities of some common essential oils. Egyptian Journal of Biology, v. 2, p. 20-27, 2000.

LACORTE, S.; FERNANDEZ-ALBA, A.R. Time of flight mass spectrometry applied to the liquid chromatographic analysis of pesticides in water and food. Mass spectrometry reviews, v. 25, n. 6, p. 866-80, 2006.

LLOYD, H.A. et al. Brunfelsamidine: a novel convulsant from the medicinal plant. Tetrahedron Letters, v. 26, n. 22, p. 2623-2624, 1985.

LONGHI, R.A. Livro das árvores: árvores e arvoretas do Sul. 2.ed. Porto Alegre: L\&PM, 1995. 176p.

LORENZI, H.; SOUZA, H.M. 2001. Plantas ornamentais no Brasil: arbustivas, herbáceas e trepadeiras. 3ed. Nova Odessa: Instituto Plantarum. 2001. 1088p.

LORENZI, H.; MATOS, F.J.A. Plantas medicinais no Brasil: nativas e exóticas. 1ed. Nova Odessa: Plantarum, 2002. 544p.

MACIEL, M.A.M. et al. Plantas medicinais: a necessidade de estudos multidisciplinares. Química Nova, v. 25, n. 3, 2002 .

MARTINS, M. B. G. et al. Caracterização anatômica, química e antibacteriana de folhas de Brunfelsia uniflora (manacá) presentes na Mata Atlântica. Revista Brasileira de Farmacognosiav. 19, n.1a, p. 106114, 2009.

MCLAUGHLIN, J.L. et al. The Use of Biological Assays to Evaluate Botanicals Drug Information Journal, n. 32, p. 513-524, 1998.

MORENO-MURILLO, B. et al. Cytotoxicity screening of some South American Solanaceae. Fitoterapia, v.72, p.680-685, 2001.

MORS, WG; Robeiro,O. Occurence of scopoletin in the genus Brunfelsia. The Journal of Organic Chemistry, v. 22, n. 8 , p. 978-979, 1957.

MORS, W.B., et al. Plant natural products against snake bite - the molecular approach. Phytochemistry, v. 55, p. 627-624, 2000.

PIO CORREAA, M. Dicionário das plantas úteis do Brasil e das exóticas cultivadas. Vol. 5. Rio de Janeiro: Ministério da Agricultura/Instituto Brasileiro de Desenvolvimento Florestal,1974. 687 p.

PLOWMAN T. Brunfelsia in Ethnomedicine. Harvard University Botanical Museum Leaflets, v. 25, p.289319,1977.

QUEIROZ, G.S. Análise de esteróides em extratos vegetais e estudo fitoquímico e biológico preliminar de Brunfelsia uniflora. Relatório (Disciplina de Estágio Supervisionado - Departamento de Química). Universidade Federal de Santa Catarina, Florianópolis,

Rev. Bras. PI. Med., Campinas, v.17, n.4, supl. III, p.1103-1111, 2015. 
2009. 45p.

RAJ R.S.N.; Radhamany P.M. Preliminary phytochemical and in vitro antioxidant properties of Brunfelsia Americana.L. Journal of Pharmaceutical Sciences and Recearch, v. 3, n. 11, p. 2712-2713, 2010.

RIBEIRO, S.R. et al. Avaliação da atividade antioxidante de Solanum paniculatum (Solanaceae). Arquivos de Ciência e Saúde UNIPAR, v.11, n.3, p.179-183, 2007.

ROCKENBACK, I.I. et. al.. Ácidos fenólicos e atividade antioxidante em fruto de Physalis peruviana L. Alimentos e Nutrição, v.19, p. 271-276, 2008.

RODOLPHO, A.D.S.; Pharmacopéia dos Estados Unidos do Brasil. 1 ed. São Paulo: Companhia Editora Nacional, 1929. 1145p

ROESLER, R. et al. Atividade antioxidante de frutas do cerrado. Ciência e Tecnologia de Alimentos Campinas, v.27, n.1, p 53-60, jan/mar 2007.

ROGINSKY, V.; LISSI, E.A. Review of methods to determine chain-breaking antioxidant activity in food. Food Chemistry, n. 92, p. 235-254, 2005.

SCHULTES, R.E. Solanaceous hallucinogens and their role in the development of New World cultures. In: HAWKES, J.G; LESTER, R.N \& SKELDING, A.D. (eds.). The Biology and Taxonomy of the Solanaceae. $1 \mathrm{ed}$. London: Academic Press, 1979. p.137-160.

SILVA, T. M. S. et. al. Studies on the alkaloids of Solanum of northeastern Brazil. Revista Brasileira de Farmacognosia, v. 15, n. 4, p. 292-293, 2005.

SIMÕES, C.M.O; SCHENKEL, E.P.; GOSMANN, G.; MELLO J.C.P de; MENTZ, L.A; PETROVICK, P.R. (Org.). Farmacognosia: da planta ao medicamento. 5 ed. Porto Alegre/Florianópolis: Editora da UFRGS / Editora UFSC, 2003. 1102p.

SOARES, S.E. Ácidos fenólicos como antioxidantes. Revista de Nutrição, v.15, n.1, p. 71-81, 2002.

SOARES, D.G. et al. Sequestering ability of butylated hydroxytoluene, propyl gallate, resveratrol, and vitamins $C$ and $E$ against ABTS, DPPH, and hydroxyl free radicals in chemical and biological systems. Journal of Agricultural and Food Chemistry, v. 51, n. 4, p. 1077-1080, 2003.

SOARES, E. L. C.; MENTZ, L. A. O Gênero Brunfelsia L. (Solanaceae) no Rio Grande do Sul, Brasil. Pesquisa, Botânica n. 58, p. 245-262, 2007.

SPADA, P.K.W.D.S.; SALVADOR, M. Antioxidant activity of the flavonoid hesperidin in chemical and biological systems. Journal of Agricultural and Food Chemistry, v. 53, n.12, p. 4757-4761, 2005.

SPAINHOUR, C.B., et al. A toxicological investigation of the garden shrub Brunfelsia calcyina var. floribunda (yesterday-today-and- tomorrow) in three species. Journal of Veterinary Diagnostic Investigation, n. 2, p. 3-8, 1990. 